\title{
UNA PRUEBA DE CUÑO EN PLOMO DE NERTOBIS
}

(1) Grup d'Investigació GRAM. Dpt. Prehistòria, Arqueologia i Hª Antiga. Universitat de València. ripolles@uv.es

(2) Investigador independiente. cores.gonzalo8@gmail.com

(3) Museu de Prehistòria de València. Diputació de València.manuel.gozalbes@dival.es

La colección de G. Cores (Madrid) conserva una singular pieza numismática de procedencia desconocida. Se trata de una placa de plomo triangular, casi isósceles, sobre la que aparece impreso el anverso de una unidad de bronce de Nertobis del tipo ACIP 1601; dos bordes parecen los originales de la plancha $(6$ y $5,40 \mathrm{~cm})$, mientras que el tercero $(5,60 \mathrm{~cm})$ es el resultado de un recorte. Sus medianas miden $5,4,5$ y $5,5 \mathrm{~cm}$, su grosor es irregular y oscila entre 6 y $10 \mathrm{~mm}$, y su peso es de $94,12 \mathrm{~g}$.

El estado de conservación es bueno, a excepción de un golpe de unos $23 \mathrm{~mm}$ en la parte superior derecha que ha deteriorado levemente la frente del retrato y ha deformado la circularidad de la gráfila. Por lo demás, la superficie del relieve es bastante nítida y con pocas corrosiones.

Sobre la plancha se estampó, con una presión bastante uniforme, un cuño circular de 31-32 mm de diámetro, con borde de perfil curvo. El resultado de la impresión es un retrato masculino imberbe, mirando a derecha, flanqueado por delfines hacia abajo y el signo ibérico $\mathbf{N}$ detrás. La figura está enmarcada por una gráfila que tiene un diámetro externo de unos 24,8 mm (espacio interior de la gráfila: $23,3 \mathrm{~mm}$ ). Desde la gráfila al borde del cuño la superficie se extiende unos $3 \mathrm{~mm}$, aproximadamente, aunque debido a que el cuño se estampó con un poco más de presión por el lado de la base del retrato, el final del cuño por esa parte es algo más amplio y queda un poco más difuminado. Todo parece indicar que el retrato y la gráfila estuvieron perfectamente centrados en la cara de impresión del cuño.

Las características de la pieza permiten afirmar que se trata de un fragmento de plancha de plomo que ha sido acuñado; la plancha ha perdido la horizontalidad de sus caras, pues en ambas se observa una cierta curvatura producida por la presión ejercida por el cuño, similar al que se produce en algunas monedas y en los ejemplares contramarcados. La nitidez del retrato es excelente, a pesar de las pequeñas concreciones y la pátina que tiene la plancha de plomo. Los detalles que muestran los rizos del peinado no respaldan la posibilidad de que haya sido producida a partir de una moneda.

Los elementos del diseño corresponden a la ceca de Nertobis. El estudio de los retratos de las emisiones de esta ceca ha permitido identificar unidades de bronce cuyo grabado es idéntico al de la plancha, lo cual permite plantear que esta última sea una prueba de cuño, realizada una vez finalizó su grabado o simplemente una impresión 


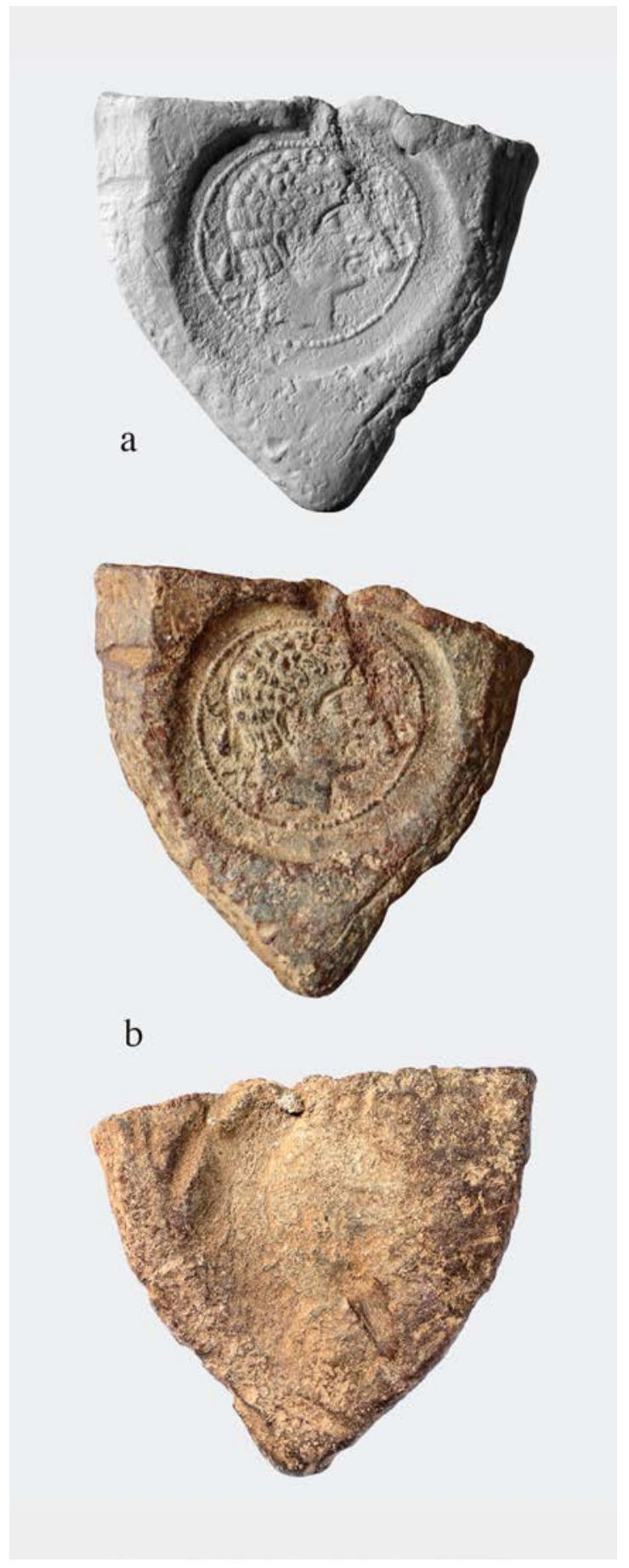

Fig. 1: Plancha de plomo con la impresión de un cuño de anverso de una unidad de bronce de Nertobis (a. Vaciado de escayola, b. Foto directa de la cara superior y la inferior). Fotos: M. C. Cores y P. P. Ripollès. realizada con el cuño original, si se prescinde de la idea de prueba o test. El mismo troquel que estampó el retrato en la plancha de plomo fue utilizado para acuñar monedas de la ciudad de Nertobis, del tipo Vives 50/1 (= CNH 244/1 y ACIP 1601). De esta emisión se conocen dos cuños de anverso y seis de reverso (fig. 2). Respecto a los anversos, uno es el de la moneda que ilustra Vives 50/1 (fig. 2, 7b) y el otro el utilizado por Villaronga para $\mathrm{CNH}$ 244/1 y ACIP 1601 (fig. 2, 4b); ambos están relacionados, ya que en el transcurso de la emisión utilizaron un mismo cuño de reverso (fig. 2, 6a, 7a, 7b y 7c). El cuño que se estampó en la plancha de plomo corresponde al de monedas similares a CNH 244/1 y ACIP 1601 (fig. 2, 4b).

Conocemos nueve monedas batidas con el cuño que fue estampado sobre la plancha de plomo. Las piezas fabricadas con este cuño tienen un diámetro que oscila entre 24 y $28 \mathrm{~mm}$. Los retratos están centrados en su mayor parte, indicando que la acuñación fue cuidadosa, no obstante los cospeles tuvieron en ocasiones un diámetro insuficiente para que pudieran grabarse el retrato y la gráfila en su integridad. En la mayor parte de las monedas la gráfila queda totalmente fuera del cospel, en unas pocas resulta parcialmente visible y en ninguna está completa. Este hecho es importante tenerlo en cuenta a la hora de valorar ante qué tipo de objeto nos encontramos. Teniendo en cuenta que en la plancha de plomo el diseño está completo, incluyendo un espacio liso entre la gráfila y el borde del cuño, no es probable que deba considerarse como una fantasía generada a partir de una moneda. Hemos de admitir que se trata de una impresión hecha con un cuño original, circunstancia que se verificaría con una acuñación centrada sobre un cospel de 31-32 mm, algo que no resulta posible, pues no se conocen monedas con un diámetro mayor de $28 \mathrm{~mm}$. Si se tiene en cuenta que la gráfila tiene un diámetro de $24,8 \mathrm{~mm}$ y que los cospeles de las monedas documentadas miden $24-28 \mathrm{~mm}$ de diámetro, se comprende la dificultad para obtener monedas centradas que incluyesen el diseño completo y la gráfila.

Su estudio nos ha llevado a consultar la bibliografía relacionada con este tipo de materiales, comprobando hasta qué punto existen valoraciones diferentes de las piezas de plomo que contienen, sobre una o dos caras, estampaciones de tipos monetarios. Los dos trabajos más completos y recientes que se han publicado sobre esta cuestión (con una amplia bibliografía) corresponden a Fischer-Bossert (2002), con motivo de la publicación de un plomo monetiforme de Siracusa, y a de Callataÿ (2010), quien desarrolla una revisión crítica sobre el 


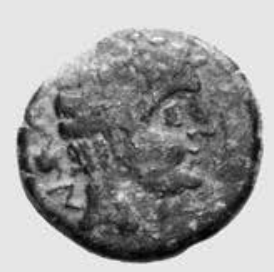

1a
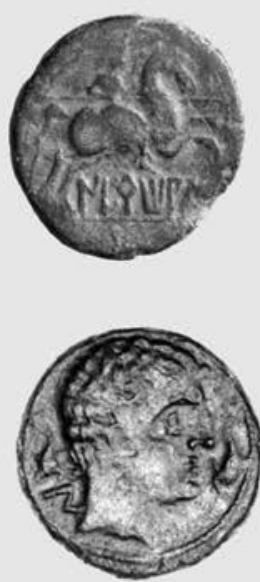

$3 a$
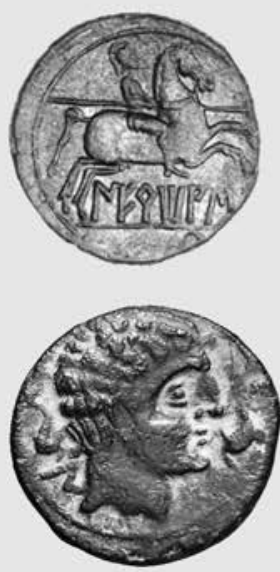

6a

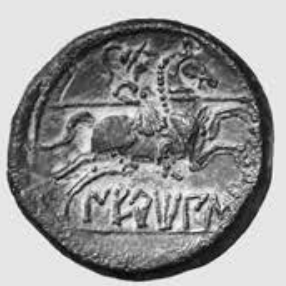

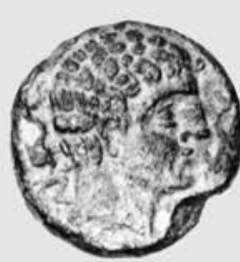

$1 \mathrm{~b}$
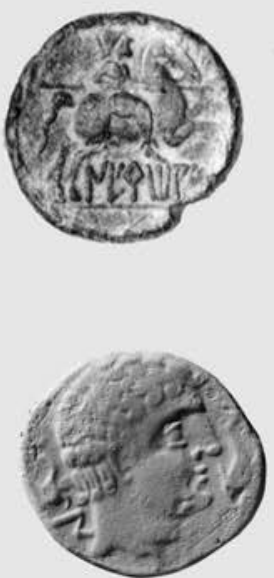

$4 \mathrm{a}$
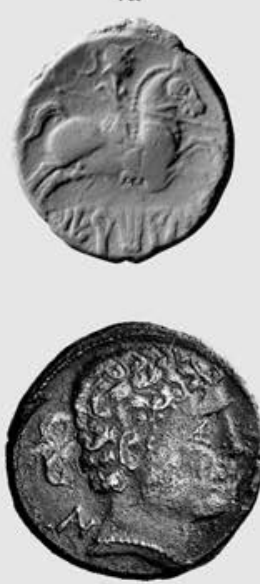

$7 \mathrm{a}$

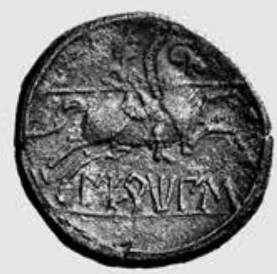

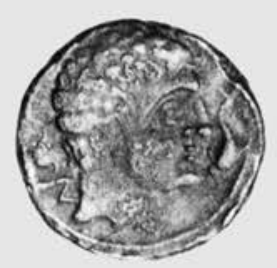

$2 \mathrm{a}$
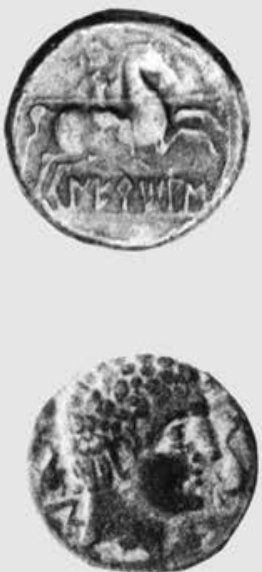

$4 \mathrm{~b}$
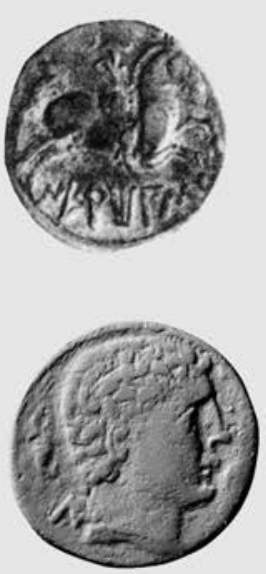

$7 b$

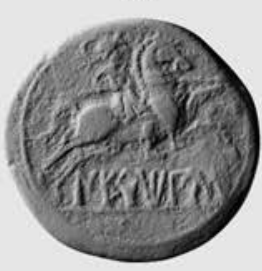

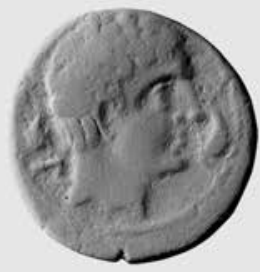

$2 b$
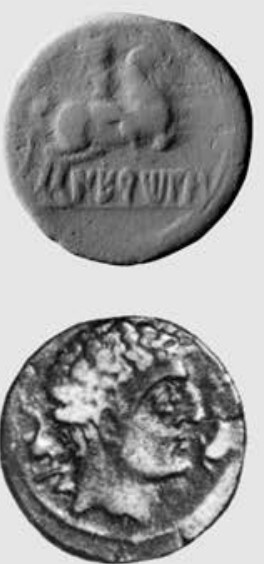

$5 a$
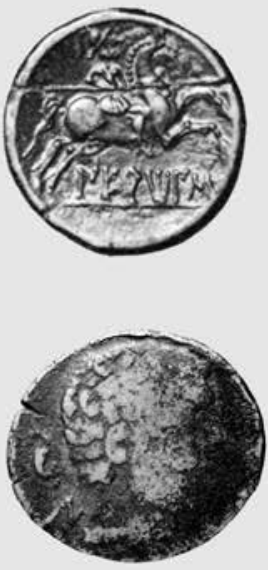

$7 \mathrm{c}$

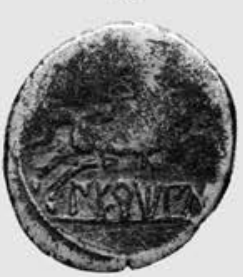

Fig. 2: Catálogo de monedas de la primera emisión de Nertobis. Las piezas 1-6 han sido acuñadas con el mismo troquel aplicado sobre la plancha de plomo. Procedencia de las piezas indicada en el catálogo. 
concepto "prueba de cuño". Fischer-Bossert es receptivo a la idea de que un buen número de testimonios corresponden a pruebas de los cuños originales o modelos de diseños para uso propio del taller, obtenido a partir de cuños originales. Por el contrario, de Callataÿ es muy crítico con la posibilidad de que estas piezas sean realmente pruebas, rechazando las teorías de Fischer-Bossert (2002: 235-239) y otros autores, y optando por catalogar la mayor parte de ellas como fantasías. No es este el lugar para valorar este tipo de materiales en su conjunto, aunque somos partidarios de mantener un espíritu crítico hacia este tipo de objetos y desde esa perspectiva hemos abordado esta nota. Como ejemplo del escepticismo frente a este tipo de piezas cabe referir un plomo que presenta el reverso del tipo RPC 271 de Celsa. Las significativas diferencias entre los diseños del plomo y las monedas impiden referirse a dicha pieza como una prueba de cuño (Collado y Gozalbes 1996).

Para el presente plomo hemos optado por la hipótesis de que se trate de una prueba de cuño. Para ello, nos apoyamos en que cumple las cuatro condiciones que debe reunir una pieza de este tipo según de Callataÿ (2010: 232). La primera condición es que el tipo se haya impreso con cuños originales; es decir, se han de descartar las piezas fundidas o las que hayan sido impresas con cuños fundidos a partir de una moneda. No hay duda que esto se cumple, pues así lo demuestran el estado de la plancha, la centralidad del diseño y la gráfila o la corona exterior.

La segunda condición que establece de Callataÿ es que la impresión en el plomo se corresponda con tipos monetarios conocidos. En nuestro caso hemos demostrado que existen monedas cuyo grabado coincide con exactitud con anversos de unidades de bronce de Nertobis.

La plancha de Nertobis también supera la tercera condición. De Callataÿ propuso que la impresión sobre plomo debe mostrar un estado de conservación mejor que el de las monedas acuñadas o en todo caso anterior a la degradación que hubiera podido tener el cuño por uso. A pesar de que el plomo es un material que se deforma con facilidad y que el presente plomo no ha sido restaurado ni limpiado, no cabe la menor duda que son visibles los detalles del peinado, incluso en las partes con más relieve de la cabeza, con tanto o incluso más detalle que el que se aprecia en las monedas mejor conservadas.

La cuarta condición establecida por de Callataÿ estipula que la prueba haya sido realizada con un cuño en preparación (en nuestro caso terminado) y que los relieves sean compatibles con los de un cuño, señalando que hay que prestar atención a todo aquello que está más allá del tipo y de la gráfila si la hay. En nuestro caso, los relieves de la plancha de plomo y los de las monedas son similares y las medidas también; además, el troquel utilizado, según se deduce de su impresión sobre el plomo, dispone de una contorno liso de ancho regular $(3 \mathrm{~mm})$.

De Callataÿ concluye que los plomos monetiformes comentados y analizados como ejemplos de pruebas de cuño incumplen todos los requisitos que permitirían identificarlos como tales; en consecuencia, propone desmantelar esta opción, que en los últimos tiempos ha tenido muchos adeptos y denominarlos "piezas de fantasía". Si bien estamos de acuerdo en ser muy rigurosos y críticos a la hora del estudio de estos materiales, creemos que no se debe descartar la idea de su existencia de modo categórico, pues la pieza que ahora presentamos reúne todas las características para considerarse como una prueba de cuño.

Son diversas las funciones que se han atribuido a las impresiones de cuños monetarios sobre plomo. En determinados casos pudieron servir como pruebas asequibles para un grabador que, sin la ayuda de acuñadores, podía comprobar el relieve y calidad de su diseño sobre un material duro antes de comenzar la producción monetaria. El hecho de que no sean habituales permite pensar que pudieron ser productos ocasionales. Por otro lado no se puede descartar que sirviesen como muestrario de diseños y capacidades en algún taller, en cuyo caso el cuño utilizado para estampar el plomo monetario pudo haber estado ya en uso (Fischer-Bossert 2002: 8). En el caso del plomo de Nertobis, nos inclinamos por describirlo como una prueba de cuño del grabador para comprobar el resultado final del trabajo.

\section{CATÁlOgO DE MONEDAS DE LA PRIMERA EMISIÓN DE NERTOBIS}

(Vives 50/1, CNH 244/1 y ACIP 1601) (fig. 2)

\section{Anverso 1 - Reverso 1}

a. Martí Hervera 20/12/2011, lote 2253, 12,30 g. b. Aureo 21/10/1997, lote 496 (= Vico 8/7/1999, lote 86), 12,40 g.

\section{Anverso 1 - Reverso 2}

a. Aureo 21/5/1998, lote 387, 13,97 g.

b. Vidal Quadras Ramón 890 (The British Museum cast collection). 
3. Anverso 1 - Reverso 3

a. Vico 11/6/1998, lote 32 (= Aureo 9/4/2003, lote $1145=21 / 1 / 2004$, lote 283$), 12,04 \mathrm{~g}$.

4. Anverso 1 - Reverso 4

a. Madrid. Col. G. Cores (= Vico 10/11/2005, lote 1134 = Cores \& Cores 2017: 228, $\left.\mathrm{n}^{\circ} 2260\right), 12,01 \mathrm{~g}, 27 \mathrm{~mm}$.

b. Col. privada $(=C N H 244 / 1=A C I P$ 1601 $)$.

5. Anverso 1 (¿evolucionado?) - Reverso 5 a. Calicó 18-19/6/1979, lote 990, 10,65 g.

6. Anverso 1 (¿evolucionado?) - Reverso 6 a. Aureo \& Calicó 26/10/2010, lote 1149, 11,24 g.

7. Anverso 2 - Reverso 6

a.Vico_2012_10_09, lote 332, ex HSA 24435, 12,39 g. b. Ex col. Jordana (= Vives 1924: lámina 50/1).

c. París. Bibliothèque nationale de France, Fonds général 465 (= Ripollès 2005: no $1186=$ http://catalogue.bnf.fr/ark:/12148/cb41748865c), 12,22 g.

\section{BIBLIOGRAFÍA}

$A C I P=$ VILLARONGA, L.; BENAGES, J. (2011): Ancient Coinage of the Iberian Peninsula / Les Monedes de l'Edat Antiga a la Peninsula Ibèrica, Barcelona.

CNH = VILLARONGA, L. (1994): Corpus Nummum Hispaniae ante Augusti Aetatem, Madrid.

COLLADO, E.; GOZALBES, M. (1996): Un plomo inédito de Celsa, Gaceta Numismática 122, 47-51.

CORES URÍA, G.; CORES GOMENDIO, M. C. (2017): Colección Cores. Moneda Antigua de la Península Ibérica. Parte I, Madrid-València.

DE CALLATAŸ, F. (2010): Les plombs à types monétaires en Grèce ancienne : monnaies (officielles, votives ou contrefaites), jetons, sceaux, poids, épreuves ou fantaisies ?, $R N$ 166, 219-255.

FISCHER-BOSSERT, W. (2002): A Lead Test-Piece of a Syracusan Tetradrachm by the Engravers Euth... and Eum..., NC 162, 1-9, pl. 1-3.

RIPOLLÈS. P. P. (2005): Monedas hispánicas de la Bibliothèque nationale de France, Madrid.

$R P C=$ BURNETT, A.; AMANDRY, M.; RIPOLLÈS, P. P. (1992): Roman Provincial Coinage, London-Paris.

Vives = VIVES Y ESCUDERO, A. (1924): La moneda hispánica, Madrid. 